\title{
Ambiente Familiar e Rendimento Escolar de Adolescentes
}

\author{
Fénita Manuel Mahendra*,1 (D) \& Angela Helena Marin² (D) \\ ${ }^{1}$ Universidade do Vale do Rio dos Sinos, São Leopoldo, RS, Brasil \\ ${ }^{2}$ Universidade Federal do Rio Grande do Sul, Porto Alegre, RS, Brasil
}

\begin{abstract}
RESUMO - O ambiente familiar tem importante ressonância no percurso escolar dos filhos. Portanto, este estudo teve como objetivo avaliar a relação entre diferentes dimensões do ambiente familiar (coesão, conflito, expressividade, independência, assertividade, orientação cultural/intelectual, lazer, religião; organização e controle) e o rendimento escolar de adolescentes com e sem histórico de reprovação. Trata-se de um estudo correlacional e comparativo, de corte transversal e caráter quantitativo, do qual participaram 24 pais e 18 adolescentes com histórico de reprovação escolar, e 19 pais e adolescentes sem histórico de reprovação, os quais responderam a Escala de Ambiente Familiar. A análise de comparação indicou que a dimensão lazer, para os pais, e coesão, para os filhos, foram mais presentes no grupo sem repetência. Além disso, a análise de correlação revelou que a dimensão conflito foi associada ao maior número de reprovações. Conclui-se que um ambiente familiar funcional pode promover o rendimento escolar.
\end{abstract}

PALAVRAS-CHAVE: ambiente familiar, rendimento escolar, fracasso escolar, ensino fundamental, adolescentes

\section{Family Environment and School Performance of Teenagers}

\begin{abstract}
Family environment has a significant impact on the school path of children. This study aimed to evaluate the relationship between different dimensions of family environment (cohesion, conflict, expressiveness, independence, assertiveness, cultural / intellectual orientation, leisure, religion; organization and control) and school performance of adolescents with and without history of school failure. It's a correlational and comparative cross-sectional and quantitative study, in which 24 parents and 18 adolescents with a failure history participated, as also 19 parents and adolescents without the same history. They answered the Family Environment Scale. The comparison analysis indicated that leisure, in parents' assessment, and cohesion, in adolescents' assessment, were more present in the group without failure. In addition, correlation analysis revealed that when more family conflict was perceived by the adolescents; greater was the number of failures they experienced. The results suggest that an appropriate family environment can stimulate school success.
\end{abstract}

KEYWORDS: family environment, school performance, school failure, elementary school, adolescents

A repercussão do ambiente familiar no percurso escolar dos filhos tem sido indicada na literatura, especialmente quando contribui para o desenvolvimento de um melhor ou pior rendimento escolar (Cia, Barham, \& Fontaine, 2012; Shek, 2002). Tais repercussões podem ser observadas desde o ensino infantil até o médio (Sy, Gottfried, \& Gottfried, 2013). Este estudo teve como objetivo avaliar a relação entre diferentes dimensões do ambiente familiar (coesão, conflito, expressividade, independência, assertividade, orientação cultural/intelectual, lazer, religião; organização e controle) e o rendimento escolar de adolescentes com e sem histórico de reprovação.
O rendimento escolar tem sido avaliado de diferentes formas, as quais incluem notas escolares e distintos instrumentos padronizados (Mahendra \& Marin, 2015). Neste estudo, optou-se por utilizar o histórico de reprovação escolar, que está pautado nas notas como critério avaliativo (Lee Blair, 2014; Sigle-Rushton, Lyngstad, Andersen, \& Kravdal, 2014; Souza \& Batista, 2018). Portanto, os alunos considerados com baixo rendimento foram os que tinham uma ou mais reprovações.

Segundo Moos e Moos (2009), é importante refletir sobre diferentes dimensões familiares quando se quer avaliar a associação positiva ou negativa destas com

\footnotetext{
* E-mail: fenita.mahendra@gmail.com

- Submetido: 10/05/2016; Revisado: 03/07/2019; Aceito: 04/10/2019.
} 
o rendimento escolar. Para eles, o ambiente familiar é entendido como o clima decorrente das dimensões da manutenção do sistema (controle e organização), das dimensões do relacionamento interpessoal (coesão, expressividade e conflito) e das dimensões de crescimento pessoal (independência, assertividade, interesses culturais e intelectuais, lazer e religião). Nas pesquisas que contemplam o rendimento escolar, as dimensões familiares têm sido estudadas principalmente no que se refere à maneira como se manifestam nas relações pais-filhos, como será apresentado a seguir.

A dimensão controle é definida como o grau em que existem regras e procedimentos estabelecidos e/ou rígidos na condução da vida familiar (Moos \& Moos, 2009). Tal dimensão tem sido investigada principalmente em relação ao controle parental, especificamente o tipo de práticas educativas utilizadas para exercer controle. Por exemplo, o estudo realizado por Sapienza, Aznar-Farias e Silvares (2009), com 66 adolescentes de ambos os sexos de $6^{\circ}, 7^{\circ}$ e $8^{\circ}$ anos do ensino fundamental de uma escola estadual de Curitiba-PR, mostrou que os pais de adolescentes com notas mais altas tendiam a utilizar menos práticas educativas negativas, caracterizadas pela disciplina relaxada, punição e abuso físico, bem como mais práticas educativas positivas, como conversa, envolvimento e democracia na tomada de decisões, quando comparados com os pais dos adolescentes com notas mais baixas. Nessa direção, Casarin e Ramos (2007) destacaram que a rigidez excessiva pode provocar um bloqueio no processo de comunicação entre pais e filhos, uma vez que estes, ao se sentirem ameaçados, podem se isolar, o que acaba por limitar seu crescimento e sua aprendizagem, diminuindo sua motivação para estudar.

A dimensão organização, que também faz parte da manutenção do sistema familiar, é entendida como a importância dada à organização e estrutura clara no planejamento das atividades e responsabilidades atribuídas aos membros da família (Moos \& Moos, 2009). Pesquisas como a de Yang e Zhou (2008) apresentaram dados que corroboraram que a maior organização e controle presentes na família, características de algumas culturas, como a oriental, podem influenciar o rendimento escolar. Os autores apontaram, por meio de uma revisão de estudos empíricos realizados nos Estados Unidos com alunos descendentes de chineses, que o maior controle e organização familiar comuns a essas famílias podiam ser responsáveis pelo seu bom rendimento escolar. As altas expectativas, os padrões rígidos, a valorização da interdependência, dos atributos acadêmicos, da autodisciplina e o foco em fomentar o crescimento pessoal dos filhos desde cedo, ensinando habilidades de negociação e solução de problemas, foram destacados como elementos importantes. Da mesma forma, entre famílias afro-americanas, a organização, estrutura e rotina familiar foram indicadas como relevantes. $\mathrm{O}$ estudo de Budescu e Taylor (2013), realizado com 115 adolescentes com idades entre 14 e 18 anos com essa descendência, ressaltou que tais características tinham efeito moderador importante sobre as consequências negativas que a falta de recursos financeiros, presente nas famílias, acarretava para os filhos, como problemas comportamentais e baixo rendimento escolar. Os autores explicaram que a falta de organização e estrutura leva à diminuição do sentimento de autoeficácia e autorregulação e ao aumento do sentimento de desamparo e da impulsividade entre adolescentes, o que pode ser amenizado por uma rotina bem estruturada. Por outro lado, pais que prezam pela organização familiar tendem a monitorar o comportamento dos filhos, garantindo que eles tenham espaço e tempo para estudar, dando-lhes suporte acadêmico.

Contudo, a literatura não é consensual sobre tais características familiares. No estudo de Karbach, Gottschling, Spengler, Hegewald e Spinath (2013), por exemplo, alto nível de controle e estrutura, quando implicam em uma pressão excessiva, criticismo e punições, mostraram-se associados ao baixo rendimento escolar. Assim, é necessário que o controle e a monitoria parental coexistam com a afetividade para com os filhos (Dumka, Gonzales, Bonds, \& Millsap, 2008). Mesmo em relação aos alunos descendentes de chineses, os resultados do estudo de Pomerantz e Wang (2009) indicaram que uma estrutura familiar rígida, com maior organização e controle, pode levar a um pior rendimento em famílias em que há menor coesão, definida como o grau de comprometimento, envolvimento, ajuda e suporte que os membros da família dão uns aos outros (Moos \& Moos, 2009). Segundo os autores, a coesão ajuda a manter, em longo prazo, a motivação dos filhos para acatar as decisões parentais, incluindo o comprometimento com a escola, uma vez que diminui o risco de os adolescentes contrariarem os pais em função das mudanças características da fase.

Nas relações familiares em que existe um controle rígido, parece existir pouco espaço para a expressividade, dimensão familiar que é compreendida como o grau em que os membros da família são encorajados para expressarem seus sentimentos, sentindo-se à vontade e acolhidos quando o fazem (Moos \& Moos, 2009). Casarin e Ramos (2007) enfatizaram essa questão mostrando a importância do carinho e aconchego na família e afirmaram que, quando as relações são rígidas, os pais também acabam não conseguindo transmitir confiança para os filhos e compartilhar ou falar sobre seus fracassos, o que faz com que não peçam ajuda nas situações que os afligem, agravando, assim, as dificuldades que vivenciam. Dessa forma, é importante que haja na família um espaço de conversação e expressão de ideias e sentimentos por parte de todos os membros. Tal condição familiar estimula o sentimento de segurança, a autoestima e o rendimento acadêmico dos filhos (Patton, Woolley, \& Hong, 2012).

O conflito, outra das dimensões familiares consideradas, pode ser definido como a presença de ira ou raiva, assim como de brigas entre os membros da família (Moos \& Moos, 
2009). Estudos mostraram que a presença de conflitos, mesmo quando não estejam diretamente relacionados aos adolescentes, pode trazer implicações negativas para o seu rendimento escolar. A pesquisa de Ghazarian e Buehler (2008), por exemplo, realizada com 2297 adolescentes norte-americanos que responderam a instrumentos para avaliar variáveis pessoais e relacionadas ao ambiente familiar, indicou que aqueles cujas famílias apresentavam mais conflitos entre os pais tinham mais probabilidade de fracassarem na escola. Os autores sugeriram que o ambiente conflituoso é estressante, na medida em que tende a instigar um sentimento de culpa nos adolescentes pelas brigas dos pais, fazendo com que sintam medo do que pode acontecer com todos os envolvidos.

Os conflitos mãe-adolescente também foram salientados no estudo de Vargas, Roosa, Knight e O’Donnell (2013), que teve como objetivo avaliar as relações existentes entre a instabilidade familiar (divórcio, separação, mudanças constantes de casa, dificuldades financeiras) e o rendimento escolar, analisando variáveis moderadoras dessa relação. A pesquisa foi realizada com 740 adolescentes americanos de origem mexicana e seus resultados mostraram que a instabilidade familiar estava positivamente associada ao conflito mãe-adolescente e este, por sua vez, estava negativamente associado à autoeficácia, comprometimento com a escola e rendimento escolar do adolescente.

Outra dimensão que Moos e Moos (2009) incluem na definição de ambiente familiar, relativa ao crescimento pessoal, é a independência, entendida como o grau em que os membros da família são autossuficientes, autônomos, defendem seus próprios direitos e tomam decisões. No estudo de Wong (2008), 171 adolescentes responderam a instrumentos que forneciam informações sobre a percepção do envolvimento parental, entendido como a disponibilidade dos pais para com os filhos, conhecimento sobre o que se passa nas suas vidas e implicação nas suas atividades diárias, além de avaliarem suporte para a autonomia e rendimento escolar. Os resultados indicaram associação positiva entre todas as variáveis consideradas.

Ainda quanto à autonomia, Wang, Chan e Lin (2012) destacaram, no seu estudo, que, quando os adolescentes tinham bom rendimento escolar, os pais incentivavam maior autonomia nos seus filhos e impunham menor controle psicológico. Já quando os adolescentes não iam bem na escola, os pais tornavam-se mais controladores, independentemente dos seus valores individuais, indicando que não somente o ambiente familiar pode interferir no rendimento escolar dos adolescentes, como também este pode influenciar as características do ambiente familiar.

A dimensão da assertividade, por sua vez, é delineada como o nível em que a competitividade e o perfeccionismo para atingir metas estão implícitos nas atividades realizadas pelos membros da família em contextos como o trabalho e a escola (Moos \& Moos, 2009). Essa dimensão familiar tem sido pesquisada em relação à ênfase que os pais colocam nas metas educacionais que têm para seus filhos, que podem influenciar suas expectativas e rendimento escolar (Bowen, Hopson, Rose, \& Glennie, 2012; Rimkute, Hirvonen, Tolvanen, Aunola, \& Nurmi, 2012). Baharudin, Hong, Lim e Zulkefly (2010) explicaram que as aspirações dos pais sobre a escolarização dos filhos refletem-se no seu envolvimento e maior monitoria em relação a assuntos escolares, tendo uma associação significativa e positiva com o rendimento escolar.

A dimensão dos interesses intelectuais e culturais tem sido pouco pesquisada em relação ao rendimento acadêmico. Ela é definida como o nível de interesse por atividades e assuntos políticos, intelectuais e culturais (Moos \& Moos, 2009). O estudo de Cano e Cardelle-Elawar (2008) mostrou que as atividades culturais e intelectuais desenvolvidas na família podem definir o perfil dos adolescentes na escola e a maneira como eles absorvem e buscam conhecimentos complementares. Participaram desse estudo 870 alunos espanhóis do ensino médio, com idades entre 12 a 18 anos, que responderam a instrumentos padronizados que avaliavam o nível educacional dos pais, o clima intelectual da família, as estratégias utilizadas pelos adolescentes no processo de aprendizagem e as crenças em relação à rapidez e ao esforço envolvidos na aprendizagem. As análises estatísticas revelaram que, quanto mais baixo o nível educacional dos pais, maior era a probabilidade de os filhos desenvolverem crenças equivocadas sobre a possibilidade de aprendizagem rápida e sem esforço e de utilizarem estratégias como memorização e reprodução acurada, com efeito negativo sobre o rendimento escolar. Entretanto, quanto maior o interesse intelectual e cultural da família, mais sofisticadas eram as crenças dos filhos, os quais tendiam a criar estratégias significativas de aprendizagem, como integração do conhecimento formal com experiência pessoal e criação de estratégias cognitivas de regulação e controle da própria aprendizagem.

Ainda em relação às dimensões de crescimento pessoal, a realização de atividades de lazer em família refere-se à quantidade de tempo despendido na participação em atividades sociais e recreacionais (Moos \& Moos, 2009) e tem sido relacionada ao ajustamento, bem-estar e desenvolvimento emocional dos adolescentes (Buswell, Zabriskie, Lundberg, \& Hawkins, 2012; Offer, 2013). Segundo Moos e Moos (2009), o baixo nível de orientação para o lazer/atividades recreacionais na família pode estar associado ao baixo rendimento escolar. As famílias que desconsideram tais atividades acabam fazendo com que seus filhos passem o tempo livre em casa, o que pode comprometer o desenvolvimento das habilidades sociais, trazendo prejuízos para o rendimento escolar.

Por fim, a dimensão da religiosidade refere-se à ênfase que é dada às questões e aos valores éticos e religiosos na família (Moos \& Moos, 2009). Fagan (2010), na sua revisão de estudos empíricos sobre a relação entre a religiosidade e o rendimento escolar, encontrou que o fato de uma família ter uma religião traz benefícios para o rendimento escolar 
dos seus filhos. Esse efeito se mostra mais significativo entre alunos de níveis socioeconômicos mais baixos, provavelmente porque para estes, a religião é uma influência positiva que pode promover a resiliência frente a situações de dificuldade ou risco. De acordo com o autor, a religiosidade contribui para a internalização de valores e normas, construindo um senso de moralidade, assim como disciplina e hábitos que regulam atividades acadêmicas e de lazer, diminuindo a tendência para comportamentos de risco. Os filhos de pais religiosos tendem a saber quais são as expectativas parentais e que eles ficariam decepcionados caso se envolvessem em comportamentos desviantes e negligenciassem seu rendimento escolar. Além disso, os adolescentes podem encontrar redes de apoio suplementares na igreja, estando rodeados de modelos e mentores que se preocupam com a educação.

No estudo de Sumari, Hussin e Siraj (2010), realizado na Malásia, para o qual foram entrevistados 20 pais de alunos com rendimento acima da média, com o objetivo de analisar a sua percepção quanto aos fatores que contribuem para esse bom rendimento, a religiosidade também foi apontada como um determinante da moralidade e do sucesso escolar dos filhos. Da mesma forma, o estudo de Park e Bonner (2008), realizado com 10.761 adolescentes americanos, teve como objetivo avaliar a associação entre o envolvimento e a afiliação religiosa da família, as práticas parentais e o rendimento escolar dos adolescentes. O rendimento escolar foi mensurado através das avaliações em matemática e na leitura e dos problemas comportamentais apresentados na escola. Os dados indicaram associação positiva entre o envolvimento religioso da família, as práticas parentais orientadas para o acompanhamento acadêmico dos filhos e o rendimento destes na leitura, assim como associação negativa com problemas de comportamento na escola. Tanto Fagan (2010) como Park e Bonner (2008) enfatizaram o papel da igreja como uma influência social orientada para a educação e a religiosidade, como uma dimensão que faz com que os membros da família passem mais tempo juntos, melhorando a coesão familiar. Moos e Moos (2009) corroboram que a ênfase na religiosidade-moralidade e no controle, combinada com maior nível de coesão e orientação para a independência em um ambiente relativamente estruturado, pode estar associada a alto rendimento escolar.

Como visto, o ambiente familiar se relaciona com o rendimento escolar de adolescentes e é importante analisá-lo tendo em consideração as diferentes dimensões que foram abordadas para que se possa adquirir um entendimento amplo e profundo do mesmo. Compreender como as relações que se estabelecem entre os membros de uma família contribuem para o baixo ou alto rendimento escolar torna-se fundamental para respaldar e validar ações de prevenção e promoção do sucesso na escola. Além disso, o foco na adolescência permite explorar essa temática centrando-se em uma fase de grandes mudanças para o indivíduo e seus familiares, principalmente no que se refere às relações entre pais e filhos, além de ser uma das fases mais difíceis de ser vivenciada no contexto escolar, pois as mudanças presentes podem levar à falta de motivação para estudar e a problemas comportamentais na escola (Cardoso \& Malbergier, 2014).

Frente ao exposto, o presente estudo buscou informações sobre o ambiente familiar considerando a perspectiva dos pais e dos filhos. Teve-se como objetivo avaliar a relação entre as dimensões do ambiente familiar (coesão familiar, conflito, expressividade, independência, assertividade, interesses culturais/intelectuais e de lazer, religião e nível de organização e controle) e o rendimento escolar de adolescentes matriculados em escolas municipais da rede fundamental de ensino de São Leopoldo - RS, comparando o perfil apresentado pelas famílias de adolescentes com e sem histórico de reprovação escolar.

\section{MÉTODO}

\section{Delineamento}

Este estudo se caracteriza como comparativo, correlacional e transversal, de caráter quantitativo (Gil, 2008; Sampieri, Collado, \& Lucio, 2013). Estudos comparativos têm como finalidade investigar indivíduos, classes, fenômenos ou fatos, com vistas a ressaltar as diferenças e similaridades entre eles, possibilitando a comparação entre agrupamentos (Gil, 2008), enquanto os estudos correlacionais visam avaliar o grau de associação entre duas ou mais variáveis, quantificando e analisando a sua vinculação (Sampieri et al., 2013).

1 A análise realizada através do Teste Qui-Quadrado mostrou que não havia diferença entre o grupo de pais que concordou em participar da

\section{Participantes}

Foram convidados a participar da amostra, de característica não probabilística e selecionada pelo critério de conveniência, todos os pais, mães ou responsáveis legais $(N=129)$ de adolescentes de 12 a 18 anos (Brasil, 1990), matriculados em duas escolas da rede municipal da cidade de São Leopoldo - RS. Contudo, apenas 43 aceitaram o convite, dos quais 24 eram pais de adolescentes que tinham histórico de uma ou mais reprovações escolares (Grupo CR), e 19 eram pais e adolescentes sem o mesmo histórico ${ }^{1}$ (Grupo SR).

pesquisa e o que não concordou, quanto ao número de reprovações de seus filhos $(p<0,62)$. 
No Grupo CR, as mães tinham, em média, 53,7 anos ( $D P$ $=17,11)$ e os pais, 44,6 anos $(D P=8,94)$. Já os adolescentes tinham idade média de 14,9 anos $(D P=1,16), 63 \%$ eram meninos e $58,3 \%$ estavam no $9^{\circ}$ ano; $20,8 \%$, no $8^{\circ}$ ano; $12,5 \%$, no $7^{\circ}$ ano; e $8,4 \%$ no $6^{\circ}$ ano. Quanto ao número de reprovações, $66,7 \%$ apresentaram uma reprovação; $25 \%$, duas; e $8,3 \%$, três. No Grupo SR, as mães tinham, em média, 39,8 anos $(D P=6,33)$ e os pais, 43,9 anos $(D P=6,34)$. Entre os adolescentes, a idade média foi de 12,7 anos $(D P=$ 1,37 ), $54 \%$ eram meninas e $36,8 \%$ estavam no $9^{\circ}$ ano; $5,3 \%$, no $8^{\circ}$ ano; $36,8 \%$, no $7^{\circ}$ ano; e $21,1 \%$, no $6^{\circ}$ ano.

Quanto à configuração familiar, no Grupo CR, 70,8\% das famílias eram nucleares, $16,7 \%$ eram monoparentais e $12,5 \%$ não informaram. No Grupo SR, $57,9 \%$ das famílias eram nucleares, $26,3 \%$ eram monoparentais e $15,8 \%$ não informaram. Em relação ao nível socioeconômico (NSE), que foi calculado com base no nível educacional e ocupacional das mães e dos pais (Hollingshead, 1975, adaptado por Tudge \& Frizzo, 2002), 88\% das famílias do grupo CR se encontravam no nível baixo ou médio-baixo e $12 \%$ não forneceram essas informações. No grupo SR, $74 \%$ pertenciam ao nível baixo ou médio-baixo; $11 \%$, ao nível médio e $15 \%$ não forneceram essas informações. Destaca-se que Ribas, Seidl de Moura e Bornstein (2003) apresentaram indicadores que apoiam a validade do Hollingshead no contexto brasileiro ao compará-lo a outras estratégias comumente utilizadas para o cálculo do nível socioeconômico.

Por fim, destaca-se que o potencial intelectual dos adolescentes, avaliado pelo Teste de Matrizes Progressivas de Raven (Angelini, Alves, Custódio, Duarte, \& Duarte, 1999), constituiu-se como um critério de inclusão-exclusão para esta amostra, sendo aplicado como primeiro instrumento da coleta de dados. Nenhum dos adolescentes considerados obteve classificação abaixo da média e, portanto, não houve exclusões.

\section{Instrumentos}

Questionário de Dados Sociodemográficos (GIDEP/ NUDIF, 2008). Instrumento utilizado para obtenção de dados do adolescente, como idade e número de reprovações escolares, bem como idade, estado civil, escolaridade e tipo de trabalho dos pais, além da configuração familiar.

Escala de Ambiente Familiar - Family Environment Scale (FES; Moos \& Moos, 2009). Escala composta de 90 afirmativas, que devem ser identificadas como verdadeiras ou falsas e que contemplam dez subescalas, chamadas de dimensões familiares (1. coesão, 2. conflito, 3. expressividade, 4. independência, 5. assertividade, 6. orientação cultural/intelectual, 7. lazer, 8. religião; 9. organização e 10. controle), que avaliam o clima social e interpessoal da família atual (forma R), idealizada (forma I) e esperada (forma E). Para fins deste estudo, apenas a Forma $\mathrm{R}$ da escala foi usada. Para análise do instrumento, obtém-se um escore bruto a partir do número de respostas relativas à cada dimensão investigada, que indicam maior ou menor presença de cada uma delas na família. Obtém-se um escore padronizado que irá indicar o perfil do ambiente familiar. Esse instrumento obteve boas propriedades psicométricas de validade e de fidedignidade para amostras brasileiras, tendo a confiabilidade da escala, avaliada pelo alfa de Cronbach, variado entre 0,61 e 0,78 para as dez subescalas (Vianna, Silva, \& Souza-Formigoni, 2007). Vem sendo utilizado por muitos estudos no país (Nader et al., 2013; Pheula, Rohde, \& Schmitz, 2011; Sherman, Duarte, \& Verdeli, 2011).

\section{Procedimentos Éticos e de Coleta}

O presente estudo faz parte de um projeto de pesquisa maior ${ }^{2}$, o qual foi avaliado e aprovado pelo Comitê de Ética em Pesquisa com Seres Humanos da Universidade do Vale do Rio dos Sinos (CAAE: 05621312.0.0000.5344). Todos os participantes foram informados a respeito dos objetivos e procedimentos da pesquisa, podendo obter qualquer esclarecimento quando desejassem. A privacidade e a confidencialidade, assim como os demais cuidados éticos, foram asseguradas.

Inicialmente, foi realizado o contato com a Secretaria Municipal de Educação de São Leopoldo - RS para apresentar a proposta do estudo e, tendo sido obtida a sua autorização, duas escolas foram contatadas para apresentação da pesquisa. As escolas contempladas foram indicadas pela Supervisão Pedagógica da Secretaria Municipal de Educação (Pedagógico/SMED), devido a terem solicitado auxílio frente às sucessivas reprovações por parte de seus alunos. Após o aceite, foi assinada a Carta de Anuência pelas direções das escolas. Na sequência, a proposta da pesquisa foi apresentada aos pais ou responsáveis legais e aos adolescentes, e o Termo de Consentimento Livre e Esclarecido, assim como o Termo de Assentimento, foram assinados por aqueles que aceitaram participar. Os pais/ responsáveis ainda responderam a um Questionário de Dados Sociodemográficos para caracterizar a família e viabilizar o contato posterior.

Para fins deste estudo, os pais foram contatados por telefone para agendar uma visita domiciliar ou um encontro na escola, durante o qual foi preenchida a Escala de Ambiente Familiar individualmente. Os adolescentes, por sua vez, eram convidados a preencher esta escala em sala de aula, em momento apropriado que foi indicado pelos professores. O tempo dispendido para aplicação foi de, aproximadamente, 30 minutos. Os alunos que não quiseram responder ao instrumento de pesquisa foram orientados a devolvê-los em branco.

2 Pesquisa intitulada "Fracasso escolar: Sintoma e/ou reatividade ao sistema de ensino?", coordenada pela Profa. Dra. Angela Helena Marin e financiado pelo CNPq. 


\section{Procedimentos de Análise de Dados}

Para a análise dos dados, foram realizadas análises descritivas para os Grupos CR e SR, considerando a avaliação dos pais e dos filhos, bem como o teste quiquadrado, para avaliação de possíveis diferenças entre os dados sociodemográficos da amostra. Após, as médias dos grupos foram comparadas quanto a cada subescala por meio do teste não-paramétrico de Mann-Whitney, uma vez que a amostra não apresentou uma distribuição normal, avaliada através do teste de Shapiro-Wilk.
Em um segundo momento, com o objetivo de analisar o grau de concordância entre os pais e filhos quanto à percepção do ambiente familiar, comparou-se o escore das subescalas do pai/mãe/responsável com o escore do filho/a por meio do Teste de Wilcoxon para medidas emparelhadas. Por fim, a análise de Correlação de Spearman foi utilizada com o objetivo de verificar a relação entre o número de reprovações dos adolescentes e as dimensões do ambiente familiar avaliadas. Os dados foram analisados no programa estatístico SPSS 22.0, considerando o nível de significância de $p<0,05$.

\section{RESULTADOS}

O Teste Qui-Quadrado revelou que não havia diferença significativa entre os Grupos CR e SR quanto ao tipo de configuração familiar $(p<0,66)$ e ao NSE, considerando o grupo de famílias de nível baixo, médio-baixo e médio $(p<$ $0,21)$, o que sugere maior uniformidade entre eles. Na Tabela 1, são apresentados os resultados das análises descritivas dos grupos CR e SR. Os resultados do Teste de Mann Whitney, realizado a fim de comparar os grupos quanto às dimensões do ambiente familiar, consta na Tabela 2.

Como se pode verificar, houve diferença entre os grupos na dimensão lazer, de acordo com a avaliação dos pais, sendo que mais atividades recreativas e de lazer eram realizadas no Grupo SR. Já na avaliação dos adolescentes, a diferença encontrada entre os grupos foi na dimensão coesão, indicando que novamente as famílias do Grupo SR eram mais coesas.

A Tabela 3 ilustra os resultados do Teste de Wilcoxon realizado para comparar a percepção do ambiente familiar entre pais e filhos. De modo geral, houve concordância entre eles e a única dimensão que apresentou diferença foi a religião, sendo que os pais perceberam as famílias como mais religiosas que os filhos.

Tabela 1

Resultados das Análises Descritivas dos Grupos CR e SR

\begin{tabular}{|c|c|c|c|}
\hline Dimensões & Grupo & $\begin{array}{c}\text { CR } \\
\text { M (DP) }\end{array}$ & $\begin{array}{c}\text { SR } \\
\text { M (DP) }\end{array}$ \\
\hline \multirow{2}{*}{ Coesão } & Pais & $50,58(12,92)$ & $54,16(11,25)$ \\
\hline & Filhos & $46,56(07,80)$ & $53,26(13,12)$ \\
\hline \multirow{2}{*}{ Conflito } & Pais & $43,92(09,50)$ & $42,53(07,81)$ \\
\hline & Filhos & $45,33(08,64)$ & $41,68(10,87)$ \\
\hline \multirow{2}{*}{ Expressividade } & Pais & $43,00(10,42)$ & $46,53(09,25)$ \\
\hline & Filhos & $43,11(07,93)$ & $45,68(09,86)$ \\
\hline \multirow{2}{*}{ Independência } & Pais & $39,33(13,65)$ & $43,95(11,61)$ \\
\hline & Filhos & $43,67(10,36)$ & $39,53(14,86)$ \\
\hline \multirow{2}{*}{ Assertividade } & Pais & $51,08(10,69)$ & $52,58(07,05)$ \\
\hline & Filhos & $54,89(08,73)$ & $54,16(09,70)$ \\
\hline \multirow{2}{*}{ Lazer } & Pais & $40,75(09,63)$ & $51,47(12,51)$ \\
\hline & Filhos & $46,06(11,87)$ & $53,21(10,91)$ \\
\hline \multirow{2}{*}{$\begin{array}{l}\text { Orientação intelectual e } \\
\text { cultural }\end{array}$} & Pais & $44,04(09,50)$ & $48,42(10,16)$ \\
\hline & Filhos & $44,61(09,14)$ & $44,74(11,95)$ \\
\hline \multirow{2}{*}{ Religião } & Pais & $60,58(05,70)$ & $61,42(06,59)$ \\
\hline & Filhos & $56,72(08,12)$ & $58,11(09,76)$ \\
\hline \multirow{2}{*}{ Organização } & Pais & $56,08(09,21)$ & $59,16(08,24)$ \\
\hline & Filhos & $56,33(08,06)$ & $56,37(10,04)$ \\
\hline \multirow{2}{*}{ Controle } & Pais & $60,75(06,74)$ & $58,37(07,17)$ \\
\hline & Filhos & $56,78(07,13)$ & $58,26(08,88)$ \\
\hline
\end{tabular}


Quanto aos resultados da correlação de Spearman relativos à avaliação dos pais, correlações negativas se estabeleceram entre número de reprovações e lazer $(r=$ $-0,42 ; p<0,01)$. No tocante à avaliação realizada pelos adolescentes, encontraram-se correlações positivas entre número de reprovações e conflito $(r=0,33 ; p<0,05)$ e correlações negativas foram observadas entre o número de reprovações e coesão $(r=-0,42 ; p<0,05)$.

Tabela 2

Comparação entre o Grupo CR e SR quanto às Dimensões do Ambiente Familiar (Teste de Mann Whitney)

\begin{tabular}{|c|c|c|c|c|c|}
\hline $\begin{array}{l}\text { Dimensões Ambiente } \\
\text { Familiar }\end{array}$ & Grupo & Reprovação escolar & $\begin{array}{c}\text { Mediana (Intervalo } \\
\text { Interquartílico) }\end{array}$ & $\mathbf{U}$ & $\mathbf{p}$ \\
\hline \multirow{2}{*}{ Coesão } & Pais & $\begin{array}{l}\mathrm{SR}(n=19) \\
\mathrm{CR}(n=24)\end{array}$ & $\begin{array}{l}59,0(45,00-65,00) \\
52,0(45,00-59,00)\end{array}$ & 184,50 & 0,28 \\
\hline & Filhos & $\begin{array}{l}\mathrm{SR}(n=19) \\
\mathrm{CR}(n=18)\end{array}$ & $\begin{array}{l}59,0(45,00-65,00) \\
48,5(43,25-52,00)\end{array}$ & 93,00 & $0,02 *$ \\
\hline \multirow{2}{*}{ Expressividade } & Pais & $\begin{array}{l}\mathrm{SR}(n=19) \\
\mathrm{CR}(n=24)\end{array}$ & $\begin{array}{l}47,0(40,00-53,00) \\
43,5(35,50-51,50)\end{array}$ & 182,50 & 0,26 \\
\hline & Filhos & $\begin{array}{l}\mathrm{SR}(n=19) \\
\mathrm{CR}(n=18)\end{array}$ & $\begin{array}{l}47,0(40,00-53,00) \\
40,0(38,50-53,00)\end{array}$ & 138,50 & 0,31 \\
\hline \multirow{2}{*}{ Conflito } & Pais & $\begin{array}{l}\mathrm{SR}(n=19) \\
\mathrm{CR}(n=24)\end{array}$ & $\begin{array}{l}39,0(39,00-49,00) \\
44,0(39,00-49,00)\end{array}$ & 213,50 & 0,71 \\
\hline & Filhos & $\begin{array}{l}\mathrm{SR}(n=19) \\
\mathrm{CR}(n=18)\end{array}$ & $\begin{array}{l}39,0(33,00-44,00) \\
44,0(39,00-54,00)\end{array}$ & 114,50 & 0,08 \\
\hline \multirow{2}{*}{ Independência } & Pais & $\begin{array}{l}\mathrm{SR}(n=19) \\
\mathrm{CR}(n=24)\end{array}$ & $\begin{array}{l}45,0(37,00-53,00) \\
45,0(31,00-53,00)\end{array}$ & 184,50 & 0,27 \\
\hline & Filhos & $\begin{array}{l}\mathrm{SR}(n=19) \\
\mathrm{CR}(n=18)\end{array}$ & $\begin{array}{l}37,0(29,00-53,00) \\
45,0(37,00-53,00)\end{array}$ & 141,00 & 0,35 \\
\hline \multirow{2}{*}{ Assertividade } & Pais & $\begin{array}{l}\mathrm{SR}(n=19) \\
\mathrm{CR}(n=24)\end{array}$ & $\begin{array}{l}53,0(47,00-59,00) \\
53,0(42,50-59,00)\end{array}$ & 227,50 & 0,99 \\
\hline & Filhos & $\begin{array}{l}\mathrm{SR}(n=19) \\
\mathrm{CR}(n=18)\end{array}$ & $\begin{array}{l}59,0(47,00-59,00) \\
59,0(47,00-60,75)\end{array}$ & 164,50 & 0,84 \\
\hline \multirow{2}{*}{$\begin{array}{l}\text { Orientação intelectual } \\
\text { e cultural }\end{array}$} & Pais & $\begin{array}{l}\mathrm{SR}(n=19) \\
\mathrm{CR}(n=24)\end{array}$ & $\begin{array}{l}47,0(41,00-58,00) \\
44,0(36,00-52,00)\end{array}$ & 169,50 & 0,15 \\
\hline & Filhos & $\begin{array}{l}\mathrm{SR}(n=19) \\
\mathrm{CR}(n=18)\end{array}$ & $\begin{array}{l}47,0(36,00-52,00) \\
41,0(39,75-48,25)\end{array}$ & 169,00 & 0,95 \\
\hline \multirow{2}{*}{ Lazer } & Pais & $\begin{array}{l}\mathrm{SR}(n=19) \\
\mathrm{CR}(n=24)\end{array}$ & $\begin{array}{l}53,0(38,00-59,00) \\
43,0(33,00-48,00)\end{array}$ & 111,50 & $0,00^{*}$ \\
\hline & Filhos & $\begin{array}{l}\mathrm{SR}(n=19) \\
\mathrm{CR}(n=18)\end{array}$ & $\begin{array}{l}53,0(48,00-59,00) \\
45,5(33,00-59,00)\end{array}$ & 114,50 & 0,08 \\
\hline \multirow{2}{*}{ Religião } & Pais & $\begin{array}{l}\mathrm{SR}(n=19) \\
\mathrm{CR}(n=24)\end{array}$ & $\begin{array}{l}61,0(61,00-66,00) \\
61,0(57,25-66,00)\end{array}$ & 202,50 & 0,51 \\
\hline & Filhos & $\begin{array}{l}\mathrm{SR}(n=19) \\
\mathrm{CR}(n=18)\end{array}$ & $\begin{array}{l}61,0(51,00-66,00) \\
56,0(51,00-61,00)\end{array}$ & 141,50 & 0,36 \\
\hline \multirow{2}{*}{ Organização } & Pais & $\begin{array}{l}\mathrm{SR}(n=19) \\
\mathrm{CR}(n=24)\end{array}$ & $\begin{array}{l}58,0(58,00-63,00) \\
58,0(53,00-63,00)\end{array}$ & 181,50 & 0,24 \\
\hline & Filhos & $\begin{array}{l}\mathrm{SR}(n=19) \\
\mathrm{CR}(n=18)\end{array}$ & $\begin{array}{l}58,0(53,00-63,00) \\
58,0(51,75-63,00)\end{array}$ & 158,00 & 0,69 \\
\hline \multirow{2}{*}{ Controle } & Pais & $\begin{array}{l}\mathrm{SR}(n=19) \\
\mathrm{CR}(n=24)\end{array}$ & $\begin{array}{l}59,0(54,00-65,00) \\
59,0(54,00-68,75)\end{array}$ & 190,50 & 0,35 \\
\hline & Filhos & $\begin{array}{l}\mathrm{SR}(n=19) \\
\mathrm{CR}(n=18)\end{array}$ & $\begin{array}{l}54,0(54,00-65,00) \\
59,0(52,75-60,50)\end{array}$ & 162,00 & 0,78 \\
\hline
\end{tabular}

*Resultado estatisticamente significativo. 
Tabela 3

Comparação da Percepção do Ambiente Familiar entre Pais e Filhos (Teste de Wilcoxon)

\begin{tabular}{|c|c|c|c|}
\hline Dimensões & $\begin{array}{l}\text { Grupo } \\
(\mathbf{n}=37) \\
\end{array}$ & $\begin{array}{c}\text { Mediana (Intervalo } \\
\text { Interquartílico) }\end{array}$ & $\mathbf{p}$ \\
\hline \multirow{2}{*}{ Coesão } & Pais & $55(46,5-59,0)$ & \multirow{2}{*}{0,07} \\
\hline & Filhos & $52(45,0-59,0)$ & \\
\hline \multirow{2}{*}{ Expressividade } & Pais & $47(40,0-53,0)$ & \multirow{2}{*}{0,95} \\
\hline & Filhos & $47(40,0-53,0)$ & \\
\hline \multirow{2}{*}{ Conflito } & Pais & $39(39,0-49,0)$ & \multirow{2}{*}{0,49} \\
\hline & Filhos & $44(36,0-46,5)$ & \\
\hline \multirow{2}{*}{ Independência } & Pais & $45(37,0-53,0)$ & \multirow{2}{*}{0,85} \\
\hline & Filhos & $37(33,0-53,0)$ & \\
\hline \multirow{2}{*}{ Assertividade } & Pais & $53(47,0-59,0)$ & \multirow{2}{*}{0,10} \\
\hline & Filhos & $59(47,0-59,0)$ & \\
\hline \multirow{2}{*}{ Orient. intelectual e cultural } & Pais & $47(41,0-58,0)$ & \multirow{2}{*}{0,17} \\
\hline & Filhos & $41(36,0-52,0)$ & \\
\hline \multirow{2}{*}{ Lazer } & Pais & $48(38,0-53,0)$ & \multirow{2}{*}{0,12} \\
\hline & Filhos & $48(40,5-59,0)$ & \\
\hline \multirow{2}{*}{ Religião } & Pais & $61(61,0-66,0)$ & \multirow{2}{*}{$0,05^{*}$} \\
\hline & Filhos & $61(51,0-66,0)$ & \\
\hline \multirow{2}{*}{ Organização } & Pais & $58(53,0-63,0)$ & \multirow{2}{*}{0,48} \\
\hline & Filhos & $58(53,0-63,0)$ & \\
\hline \multirow{2}{*}{ Controle } & Pais & $59(54,0-65,0)$ & \multirow{2}{*}{0,24} \\
\hline & Filhos & $59(54,0-65,0)$ & \\
\hline
\end{tabular}

*Resultado estatisticamente significativo.

\section{DISCUSSÃO}

Como sabido, teve-se como objetivo avaliar a relação entre as dimensões do ambiente familiar (coesão familiar, conflito, expressividade, independência, assertividade, orientação cultural e intelectual, lazer, religião e nível de organização e controle) e o rendimento escolar de adolescentes com e sem histórico de reprovação escolar. Os resultados indicaram que a dimensão lazer foi mais presente nas famílias dos alunos sem histórico de reprovação escolar, considerando a avaliação dos pais, assim como a dimensão coesão, na avaliação dos filhos. A análise de correlação confirmou esses dados, indicando que, quanto mais lazer, na avaliação dos pais, e coesão, na avaliação dos filhos, menor o número de reprovação entre os adolescentes. Da mesma forma, quanto maior a percepção de conflito familiar por parte dos filhos, maior o número de reprovação escolar.

Tais dados corroboram a literatura que indica associação entre a participação dos adolescentes em atividades estruturadas fora da escola com melhor rendimento escolar (Bartko \& Eccles, 2003) e mais aspirações educacionais (Guest \& Schneider, 2003). A participação em atividades extracurriculares estruturadas também já se mostrou relacionada à maior satisfação com a escola (Gilman, 2001) e com menor incidência de evasão escolar (Mahoney, 2001). Segundo Knifsend e Graham (2012), o envolvimento em atividades extracurriculares realizadas na escola (grupos de liderança, atividades artísticas, clubes ou esportes) repercute positivamente no rendimento escolar por meio do provimento de diferentes contextos nos quais os adolescentes podem se relacionar com alunos e profissionais da escola e, assim, sentirem-se pertencentes a ela. A associação entre o lazer e melhor ajustamento, bem-estar e desenvolvimento emocional dos adolescentes também tem sido destacada por outros autores (Buswell et al., 2012; Offer, 2013).

Salienta-se que grande parte das famílias avaliadas neste estudo teve escore baixo na dimensão lazer, pois reportaram atividades como assistir televisão e escutar rádio como suas principais formas de diversão, item que tem pontuação negativa no instrumento de avaliação utilizado. Pesquisas anteriores mostraram que o tempo gasto em frente à televisão está negativamente associado ao rendimento escolar e que esse tipo de atividade também influencia de modo negativo a capacidade de concentração dos indivíduos (Cooper, Valentine, Nye, \& Lindsay, 1999; Esteban-Cornejo et al., 2015).

Uma reflexão importante a ser feita é sobre o difícil acesso das famílias participantes deste estudo a espaços de lazer devido a residirem em bairros de maior vulnerabilidade social. Portanto, caberia à sociedade somar esforços para 
disponibilizar espaços dessa natureza às famílias menos favorecidas economicamente. Além disso, o lazer não tem sido considerado pelas pesquisas como uma dimensão familiar, mas como um fator individual, referindo-se às atividades nas quais o adolescente investe seu tempo livre (Bartko \& Eccles, 2003; Knifsend \& Graham, 2012). Assim, estudos futuros precisam ser realizados para melhor elucidar essa associação, envolvendo a análise do tempo livre compartilhado em família em atividades dentro e fora de casa, bem como do nível socioeconômico e do contexto cultural, que muito podem influenciar na maneira como essa dimensão é vivenciada e avaliada pelas famílias.

A diferença encontrada entre os grupos na dimensão coesão e a correlação positiva entre a dimensão conflito e o número de reprovações também corroborou as informações já apontadas em outros estudos. Conforme Mohanraj e Latha (2005), quando os adolescentes vivenciam mais brigas, criticismo e discussões na família, seu rendimento escolar tende a ser mais baixo. Também Ghazarian e Buehler (2008) e Vargas et al. (2013) indicaram a associação entre um ambiente familiar conflituoso e o comprometimento do rendimento escolar na fase adolescente. Por outro lado, como mostram Bacro (2012), Bodovski e Youn (2010) e Pomerantz e Wang (2009), coesão, calorosidade, demonstração de afeto e proximidade entre os membros da família reverberam positivamente no rendimento escolar. O envolvimento parental, entendido como o sentimento de os pais estarem ao lado dos filhos e reconhecerem seus esforços, é relacionado ao desenvolvimento da autonomia, que facilita a autodeterminação e a solução de problemas, que, por sua vez, são importantes para o rendimento escolar (Shen, 2011).

No que concerne à concordância entre a percepção de pais e adolescentes quanto ao ambiente familiar, encontrou- se que os pais percebiam suas famílias como sendo mais religiosas do que seus filhos, o que pode ser explicado pelo fato de possivelmente eles valorizarem e terem maior interesse nessa dimensão. Para melhor elucidar as concordâncias ou discrepâncias do ambiente familiar entre pais e filhos, análises qualitativas podem ser realizadas com vistas a avaliar como cada uma das dimensões é percebida pelos membros da família. Assim, novos estudos que analisem tais discrepâncias de maneira a entender como elas se constituem e como podem se relacionar com os processos individuais e familiares são necessários.

Ainda, como sugestão para estudos futuros, pode-se apontar o uso de amostras maiores e representativas, contemplando famílias de diversos contextos socioeconômicos, e a utilização de análises estatísticas mais complexas, que possibilitarão encontrar novos resultados sobre as associações e elucidar quais dimensões familiares podem se comportar como determinantes e mediadoras na relação com o rendimento escolar. Também se destaca a necessidade de avaliar o rendimento escolar para além do histórico de reprovação escolar dos alunos, considerando suas notas e habilidades atuais.

Frente aos resultados encontrados, constatou-se que o ambiente familiar tem repercussões no rendimento escolar de adolescentes, especialmente no que diz respeito ao lazer, à coesão e ao conflito familiar na amostra investigada. Ressalta-se que os dados obtidos indicam a importância de avaliar o ambiente familiar considerando as suas várias dimensões, desde as intrafamiliares até a sua relação com o ambiente extrafamiliar. Além disso, eles indicaram que um ambiente familiar funcional pode promover o rendimento dos adolescentes na escola, aspecto que precisa ser considerado pelos profissionais que trabalham na área da saúde e educação com vista a intervir junto ao fenômeno.

\section{REFERÊNCIAS}

Angelini, A. L., Alves, I. C. B., Custódio, E. M., Duarte, W. F., \& Duarte, J. L. M. (1999). Manual: Matrizes Progressivas Coloridas de Raven [Manual: Raven's Coloured Progressive Matrices]. São Paulo, SP: Centro Editor de Testes e Pesquisas em Psicologia.

Bacro, F. (2012). Perceived attachment security to father, academic self-concept and school performance in language mastery. Journal of Child \& Family Studies, 21(6), 992-1002. https:// doi.org/10.1007/s10826-011-9561-1

Baharudin, R., Hong, C. Y., Lim, S. J., \& Zulkefly, N. S. (2010). Educational goals, parenting practices and adolescents' academic achievement. Asian Social Science, 6(12), 144-152.

Bartko, W. T., \& Eccles, J. S. (2003). Adolescent participation in structured and unstructured activities: A person-orientated analysis. Journal of Youth and Adolescence, 32(4), 233-242. https://doi.org/10.1023/A: 1023056425648

Bodovski, K., \& Youn, M.-J. (2010). Love, discipline and elementary school achievement: The role of family emotional climate. Social Science Research, 39(4), 585-595. https://doi. org/10.1016/j.ssresearch.2010.03.008
Bowen, G. L., Hopson, L. M., Rose, R. A., \& Glennie, E. J. (2012). Students' perceived parental school behavior expectations and their academic performance: A longitudinal analysis. Family Relations, 61, 175-91. https://doi.org/10.1111/j.17413729.2011.00695.x

Brasil. (1990). Estatuto da Criança e do Adolescente. Retrieved from http://www.crianca.mppr.mp.br/arquivos/File/publi/ camara/estatuto_crianca_adolescente_9ed.pdf

Budescu, M., \& Taylor, R. (2013). Order in the home: Family routines moderate the impact of financial hardship. Journal of Applied Developmental Psychology, 34(2) 63-72.

Buswell, L., Zabriskie, R., Lundberg, N., \& Hawkins, A. (2012). The relationship between father involvement in family leisure and family functioning: The importance of daily family leisure. Leisure Sciences, 34(2), 172-190. https://doi.org/10.1080/014 90400.2012 .652510

Cano, F., \& Cardelle-Elawar, M. (2008). Family environment, epistemological beliefs, learning strategies, and academic performance: A path analysis. In M. S. Khine (Ed.), Knowing, knowledge and beliefs: Epistemological studies across diverse cultures (pp. 219-239). New York: Springer. 
Cardoso, L. R. D., \& Malbergier, A. (2014). Problemas escolares e o consumo de álcool e outras drogas entre adolescentes. Revista Quadrimestral da Associação Brasileira de Psicologia Escolar e Educacional, 18(1), 27-34. Retrieved from http://www.scielo. br/pdf/pee/v18n1/v18n1a03.pdf

Casarin, N. E. F., \& Ramos, M. B. J. (2007). Família e aprendizagem escolar. Revista Psicopedagogia, 24(74), 182-201.

Cia, F., Barham, E. J., \& Fontaine, A. M. G. V. (2012). Desempenho acadêmico e autoconceito de escolares: Contribuições do envolvimento paterno. Estudos de Psicologia, 29(4), 461470. Retrieved from http://www.scielo.br/pdf/estpsi/v29n4/ v29n4a01.pdf

Cooper, H., Valentine, J. C., Nye, B., \& Lindsay, J. (1999). Relationships between five after-school activities and academic achievement. Journal of Educational Psychology, 91(2), 369378. https://doi.org/10.1037/0022-0663.91.2.369

Dumka, L. E., Gonzales, N. A., Bonds, D. D., \& Millsap, R. E. (2008). Academic success of Mexican origin adolescent boys and girls: The role of mothers' and fathers' parenting and cultural orientation. Sex Roles, 60, 588-599.

Esteban-Cornejo, I., Martinez-Gomez, D., Sallis, J. F., CabanasSánchez, V., Fernández-Santos, J., ... Veiga, O. L. (2015). Objectively measured and self-reported leisure-time sedentary behaviour and academic performance in youth: The UP\&DOWN Study. Preventive Medicine, 77, 106-111. https:// doi.org/10.1016/j.ypmed.2015.05.013

Fagan, P. (2010). Religious practice and educational attainment: How worship influences academic success. Retrieved from: http://www.ncfpc.org/FNC/1010S2-Fagan.pdf

Ghazarian, S. R., \& Buehler, C. (2008). Interparental conflict and academic achievement: An examination of mediating and moderating factors. Journal of Youth and Adolescence, 39(1), 23-35. https://doi.org/10.1007/s10964-008-9360-1

GIDEP/NUDIF (2008). Questionário sobre os dados sociodemográficos da família (Unpublished manuscript). Instituto de Psicologia, UFRGS, Porto Alegre, RS, Brasil.

Gil, A. C. (2008). Métodos e técnicas de pesquisa social. São Paulo, SP: Atlas.

Gilman, R. (2001). The relationship between life satisfaction, social interest, and frequency of extracurricular activities among adolescent students. Journal of Youth and Adolescence, 30(6), 749-768. https://doi.org/10.1023/A:1012285729701

Guest, A., \& Schneider, B. H. (2003). Adolescents' extracurricular participation in context: The mediating effects of schools, communities, and identity. Sociology of Education, 76(2), 89-95. https://doi.org/10.2307/3090271

Hollingshead, A. B. (1975). Four factor index of social status (Unpublished manuscript). Yale University, New Haven, Connecticut, United States of America.

Karbach, J., Gottschling, J., Spengler, M., Hegewald, K., \& Spinath, F. (2013). Parental involvement and general cognitive ability as predictors of domain-specific academic achievement in early adolescence. Learning and Instruction, 23, 43-51. https://doi. org/10.1016/j.learninstruc.2012.09.004

Knifsend, C. A., \& Graham, S. (2012). Too much of a good thing? How breadth of extracurricular participation relates to schoolrelated affect and academic outcomes during adolescence. Journal of Youth and Adolescence, 41(3), 379-389. https://doi. org/10.1007/s10964-011-9737-4

Lee Blair, S. (2014). Parental involvement and children's educational performance: A comparison of Filipino and U.S. parents. Journal of Comparative Family Studies, 45(3), 351-366. Retrieved from http://web.a.ebscohost.com/ehost/ pdfviewer/pdfviewer?sid $=820 \mathrm{cf} 975-\mathrm{a} 6 \mathrm{a} 4-42 \mathrm{~b} 0-\mathrm{bb} 2 \mathrm{c}-\mathrm{f} 2 \mathrm{fab} 2$ 0a6537\%40sessionmgr4004\&vid $=8 \&$ hid $=4204$

Mahoney, J. L. (2001). Children who participated in school extracurricular activities were less likely to drop out or to have been arrested. Evidence-Based Mental Health, 4(1), 29-30. https://doi.org/10.1136/ebmh.4.1.29

Mohanraj, R., \& Latha (2005). Perceived family environment in relation to adjustment and academic achievement. Journal of the Indian Academy of Applied Psychology, 31(1-2), 18-23. Retrieved from http://medind.nic.in/jak/t05/i1/jakt05i1p18.pdf Mahendra, F., \& Marin, A. H. (2015). Ambiente familiar e desempenho escolar: Uma revisão sistemática. Psicologia da Educação, 40, 41-57.

Moos, R. H., \& Moos, B. S. (2009). Family Environment Scale manual and sampler set: Development, applications and research (4th ed.). Palo Alto, CA: Mind Garden, Inc.

Nader, E. G., Kleinman, A., Gomes, B. C., Lafer, B., Caetano, S. C., Bruscagin, C., ... Soares, J. C. (2013). Negative expressed emotion best discriminates families with bipolar disorder children. Journal of Affective Disorders, 148, 418-423. https:// doi.org/10.1016/j.jad.2012.11.017

Offer, S. (2013). Family time activities and adolescents' emotional well-being. Journal of Marriage and Family, 75(1), 26-41. https://doi.org/10.1111/j.1741-3737.2012.01025.x

Park, H. S., \& Bonner, P. (2008). Family religious involvement, parenting practices and academic performance in adolescents. School Psychology International, 29(3), 348-362.

Patton, D. U., Woolley, M. E., \& Hong, J. S. (2012). Exposure to violence, student fear, and low academic achievement: African American males in the critical transition to high school. Children and Youth Services Review, 34(2), 388-395.

Pheula, G. F., Rohde, L. A., \& Schmitz M. (2011). Are family variables associated with ADHD, inattentive type? A casecontrol study in schools. European Child \& Adolescent Psychiatry, 20, 137-145. https://doi.org/10.1007/s00787-0110158-4

Pomerantz, E. M., \& Wang, Q. (2009). Are gains in decision-making autonomy during early adolescence beneficial for emotional functioning? The case of the United States and China. Child Development, 80(6), 1705-1721.

Ribas, R. C. Jr., Seidl de Moura, M. L., \& Bornstein, M. (2003). Socioeconomic status in Brazilian psychological research: II socioeconomic status and parenting knowledge. Estudos de Psicologia, 8, 385-392. Retrieved from http://www.scielo.br/ pdf/epsic/v8n3/19960.pdf

Rimkute, L., Hirvonen, R., Tolvanen, A., Aunola, K., \& Nurmi, J.-E. (2012). Parents' role in adolescents' educational expectations. Scandinavian Journal of Educational Research, 56(6), 571590.

Sampieri, R. H., Collado, C. F., \& Lucio, M. del P. B. (2013). Metodologia de pesquisa (5th ed.). Porto Alegre, RS: Penso.

Sapienza, G., Aznar-Farias, M., \& Silvares, E. (2009). Competência social e práticas educativas parentais em adolescentes com alto e baixo rendimento acadêmico. Psicologia: Reflexão $e$ Crítica, 22(2), 208-213.

Shek, D. T. (2002). Family functioning and psychological wellbeing, school adjustment, and problem behavior in Chinese adolescents with and without economic disadvantage. The Journal of Genetic Psychology, 163(4), 497-502. https://doi. org/10.1080/00221320209598698

Shen, Y. L. (2011). Effects of Chinese parental practices on adolescent school outcomes mediated by conformity to parents, self-esteem, and self-efficacy. International Journal of Educational Research, 50, 282-290.

Sherman, B. J., Duarte, C. S., \& Verdeli, H. (2011). Internalizing and externalizing problems in adolescents from Bahia, Brazil. Sociodemographic correlates and family environment in boys and girls. International Journal of Mental Health, 40(3), 55-76. Retrieved from http://web.a.ebscohost.com/ehost/pdfviewer/ pdfviewer?sid=820cf975-a6a4-42b0-bb2c-f2fab20a6537\%4 0sessionmgr4004\&vid=16\&hid=4204 
Sigle-Rushton, W., Lyngstad, T. H., Andersen, P. L., \& Kravdal, Ø. (2014). Proceed with caution? Parents' union dissolution and children's educational achievement. Journal of Marriage \& Family, 76(1), 161-174. https://doi.org/10.1111/jomf.12075

Souza, P. B. de, \& Batista, A. P. (2018). Desempenho acadêmico e percepção de crianças sobre estilos de liderança de professores. Psicologia Escolar e Educacional, 22(1), 3745. https://doi.org/10.1590/2175-35392018011081

Sumari, M., Hussin, Z., \& Siraj, S. (2010). Factors contributing to academic achievement and moral development: A qualitative study. The International Journal of Research and Review, $5(2), 18-23$.

Sy, S. R., Gottfried, A. W., \& Gottfried, A. E. (2013). A transactional model of parental involvement and children's achievement from early childhood through adolescence. Parenting: Science and Practice, 13(2), 133-152. https://doi.org/10.1080/152951 92.2012.709155

Tudge, J. R. H., \& Frizzo, G. F. (2002). Classificação baseada em Hollingshead do nível sócio-econômico das famílias do estudo longitudinal de Porto Alegre: Da gestação à escola (Unpublished manuscript). Instituto de Psicologia, Universidade Federal do Rio Grande do Sul, Porto Alegre, RS, Brasil.
Vargas, D. A., Roosa, M. W., Knight, G. P., \& O’Donnell, M. (2013). Family and cultural processes linking family instability to Mexican American adolescent adjustment. Journal of Family Psychology, 27(3), 387-397.

Vianna, V. P. T., Silva, E. A., \& Souza-Formigoni, M. L. (2007). Versão em português da Family Environment Scale: Aplicação e validação. Revista Saúde Pública, 41(3), 419-426. https://doi. org/10.1590/S0034-89102007000300014

Wang, Q., Chan, H., \& Lin, L. (2012). Antecedents of Chinese parents' autonomy support and psychological control: The interplay between parents' self-development socialization goals and adolescents' school performance. Journal of Youth and Adolescence, 41, 1442-1454.

Wong, M. M. (2008). Perceptions of parental involvement and autonomy support: Their relations with self-regulation, academic performance, substance use and resilience among adolescents. North American Journal of Psychology, 10(3), 497- 518.

Yang, W., \& Zhou, W. (2008). What accounts for Chinese-American children's high academic performance: A literature review of parental influences and home environment. Gifted Education International, 24(1), 88-104. 